\title{
Skeletal Muscle Deoxygenation During Exercise Assessed by Near-Infrared Spectroscopy and its Relation to Expired Gas Analysis Parameters
}

\author{
Takeshi Miura, MD; Toru Takeuchi, MD; Hiroshi Sato, MD; Naoko Nishioka, MD; \\ Setsuo Terakado, MD; Yoshiharu Fujieda, MD; Chiharu Ibukiyama, MD
}

\begin{abstract}
The present study was performed to determine the relation between oxygenated hemoglobin (oxy- $\mathrm{Hb})$ changes in working muscles and ventilatory parameters. Six active normal subjects, 21 sedentary normal subjects and 16 patients with heart failure performed an incremental exercise with expired gas analysis. Deoxygenation of the vastus lateralis muscle was monitored for oxy- $\mathrm{Hb}$ changes using near-infrared spectroscopy. Near the anaerobic threshold (AT), oxy- $\mathrm{Hb}$ started to decrease, forming the first inflection point (P1). Near the respiratory compensation point (RCP), the second inflection point (P2) was observed. Oxygen uptake at the AT, RCP, P1 and P2 decreased in magnitude first in the active normal subjects, then in sedentary normal subjects and finally in the heart failure patients. High correlation was demonstrated between AT and P1 $(r=0.8, p<0.0005)$ and between $\mathrm{RCP}$ and $\mathrm{P} 2(\mathrm{r}=0.9, \mathrm{p}<0.0005)$. In 12 sedentary normal subjects who underwent repeat exercise, reproducibility was confirmed for both P1 and P2. Constant work rate exercises were performed in 5 sedentary normal subjects, and in all of them the oxy-Hb remained unchanged below the AT work rate, whereas oxy-Hb decreased above the AT work rate. Exercise capacity, with respect to both working muscle deoxygenation and ventilation, could be evaluated in detail by the concomitant use of near-infrared spectroscopy and expired gas analysis. (Jpn CirC $J$ 1998; 62: 649-657)
\end{abstract}

Key Words: Heart failure; Expired gas analysis; Near-infrared spectroscopy; Skeletal muscle; Working capacity

$\mathbf{E}$ xercise intolerance and left ventricular dysfunction are both significant predictors of prognosis in patients with heart failure! In these patients, exercise capacity is limited by abnormalities of cardiac function, ventilation, skeletal muscle blood flow, skeletal muscle oxygen extraction, or skeletal muscle metabolism? Methods to assess blood flow and oxygen kinetics in the lower limbs have utilized femoral vein catheter techniques (including thermodilution, dye-dilution and determination of the difference between arterial and venous oxygen), 3 venous plethsmography ${ }^{4}$ and the ${ }^{133} \mathrm{Xe}$ technique? However, all these methods are difficult to apply to working muscles during exercise. Since the application of near-infrared (NIR) spectroscopy in vivo by Jöbsis, it has been used as a non-invasive method to assess the oxygen kinetics in tissues, mainly through monitoring the oxygenation and deoxygenation of hemoglobin? although this method cannot measure myoglobin and hemoglobin separately. In animal and human skeletal muscles during exercise, the changes in oxygenated hemoglobin (oxy-Hb) content measured by NIR spectroscopy bears a high correlation with the changes in venous hemoglobin oxygen saturation, and could be used to determine the oxygen kinetics in working muscles during exercise., ${ }^{8}$ Wilson et al reported greater oxy-Hb decrease in the vastus lateralis muscles in

(Received March 9, 1998; revised manuscript received May 18, 1998; accepted May 19, 1998)

Second Department of Internal Medicine, Tokyo Medical College, Tokyo, Japan

Mailing address: Takeshi Miura, MD, Second Department of Internal Medicine, Tokyo Medical College, 6-7-1, Nishi-Shinjuku, Shinjukuku, Tokyo, 160-0023, Japan heart failure patients than in sedentary normal subjects at the same work rate, and demonstrated the possible clinical applications of the method? Matsui et al reported an earlier occurrence of oxy-Hb decrease in femoral muscles in patients with heart failure compared to normal subjects! ${ }^{10}$ However, NIR spectroscopy measures the relative changes of oxy- $\mathrm{Hb}$, deoxygenated hemoglobin (deoxy- $\mathrm{Hb}$ ) and total hemoglobin volume (total-Hb) using NIR light absorption changes, and quantification of these parameters in human is difficult to achieve.

Belardinelli et al performed constant load exercise tests in normal subjects, and reported that oxy-Hb reached a constant state at a workload lower than the anaerobic threshold (AT; obtained from expired gas analysis) but decreased at a workload higher than the AT!1 ${ }^{1}$ Another study that used a ramp load in normal subjects, identified the inflection point of oxy-Hb decrease in the femoral quadriceps muscle and reported a high correlation with the AT!2 Takahashi et al performed ramp load exercise tests in athletes, normal subjects and patients with heart failure, and reported an inflection point of oxy-Hb decrease in the femoral quadriceps muscle and a high correlation with the AT!3 If an inflection point can be identified from the NIR spectroscopy measurements of oxy-Hb changes in working muscles, then quantification may be possible by simultaneously analyzing the expired gas which provides the oxygen uptake $\left(\dot{\mathrm{V}}_{2}\right)$ at specific points of oxy-Hb and deoxy-Hb changes. Although the AT and the respiratory compensation point $(\mathrm{RCP})$, which is the starting point of respiratory compensation against metabolic acidosis, can be obtained from expired gas analysis, this method involves computing the points of metabolic changes in working muscles indi- 
rectly from expired gas analysis data ${ }^{14-17}$ Even though expired gas analysis parameters such as AT and RCP are related to oxy-Hb and deoxy-Hb changes, the detailed relationship between parameters of expired gas analysis and NIR spectroscopy measurements has not been reported, and the reproducibility of the NIR spectroscopy parameters has not been assessed.

We studied 3 groups of subjects with various degrees of exercise tolerance (active normal subjects, sedentary normal subjects and patients with chronic heart failure) to investigate (1) whether it is possible to determine the inflection points for oxy-Hb and deoxy-Hb in NIR spectroscopy measurements in working muscles during exercise, (2) the reproducibility of the oxy-Hb or deoxy-Hb changes, (3) the relation between the inflection points and exercise tolerance, and (4) the relation between the inflection points and AT and RCP. Because the relationship between oxy-Hb or deoxy-Hb change and AT has only been described as a phenomenon and the mechanism has not been examined, we also investigated (5) the oxy- $\mathrm{Hb}$ and deoxy-Hb changes during the application of constant work rates at intensities below and above the AT level.

\section{Methods}

\section{Subjects}

We studied a total of 43 subjects in whom AT determination was possible from expired gas analysis. They included 6 male active normal subjects (age $23 \pm 3$ years, height $177 \pm 4 \mathrm{~cm}$, weight $68 \pm 5 \mathrm{~kg}$ ), 21 sedentary normal subjects (14 males and 7 females, age $27 \pm 4$ years, height $167 \pm 3 \mathrm{~cm}$, weight $60 \pm 8 \mathrm{~kg}$ ) and 16 patients with chronic heart failure (14 males and 2 females, age 54 \pm 9 years, height $167 \pm 7 \mathrm{~cm}$, weight $63 \pm 9 \mathrm{~kg}$ ). The body mass index of all subjects was within 28. Normal subjects were free of anemia, cardiac disease, pulmonary disease and peripheral vascular disease. All active normal subjects exercised 3 times a week, for at least $1 \mathrm{~h}$ each time. Sedentary subjects did not exercise regularly. Heart failure patients who had anemia or pulmonary or peripheral vascular diseases were excluded, and patients with ischemic heart diseases who demonstrated exercise-induced myocardial ischemia were also rejected. All patients with heart failure had a past history of heart failure symptoms. All patients in heart failure were receiving medication to control it. Exercise tests were conducted while the patients were on medication. Ten patients were on diuretics, 7 were on digitalis, 10 were on angiotensin-converting enzyme inhibitors and 8 were on other vasodilators. The underlying diseases causing heart failure included dilated cardiomyopathy in 7 patients, mitral regurgitation in 4 , aortic regurgitation in 1 , ischemic cardiomyopathy in 3 and diabetic cardiomyopathy in 1 . One patient was classified old NYHA Class I, 11 patients were Class II and 4 were Class III. The average left ventricular ejection fraction in the heart failure patients was $43 \pm 9 \%$ (range, 19-77\%). In mitral regurgitation and aortic regurgitation patients, the ejection fraction was within normal range.

Written informed consent was obtained from all subjects before the study. The study protocol was approved by the Ethical Committee of Tokyo Medical College.

\section{Near-Infrared Spectroscopy}

A near-infrared spectrophotometer Model OM-100A (Shimadzu) was used. Using surgical tape, the probe was fixed on the right lateral vastus muscle at one-third of the length from the distal end, and the tissue was irradiated with near-infrared light at 780,805 and $830 \mathrm{~nm}$. The changes in absorbance were recorded, and oxy-Hb, deoxy$\mathrm{Hb}$ and total- $\mathrm{Hb}$ values were computed to assess the oxygenation status of the muscle. The oxy-Hb, deoxy-Hb and total-Hb values were calculated from formulae determined experimentally by Tamura et al ${ }^{18}$ using human erythrocyte suspensions, and were derived from the Lambert-Beer Law and based on the principles of Hazeki et al ${ }^{19}$ and Seiyama et al? ${ }^{20}$

oxy-Hb $=-3 \Delta \mathrm{A} 805+3 \Delta \mathrm{A} 830$

deoxy-Hb $=1.6 \Delta \mathrm{A} 780-2.8 \Delta \mathrm{A} 805+1.2 \Delta \mathrm{A} 830$

total- $\mathrm{Hb}=1.6 \Delta \mathrm{A} 780-5.8 \Delta \mathrm{A} 805+4.2 \Delta \mathrm{A} 830$

where $\Delta \mathrm{A} 780, \Delta \mathrm{A} 805$ and $\Delta \mathrm{A} 830$ are the absorption changes at wavelengths 780,805 and $830 \mathrm{~nm}$.

The optical pathlength of the NIR light in tissue was evaluated by the direct method ${ }^{21}$ or using the Monte Carlo modeling.2 The average depth of measurement in the tissue has been confirmed to be half of the distance between the light guide and the detector. In the present study, the distance was set at $40 \mathrm{~mm}$, and the measurement average depth was approximately $20 \mathrm{~mm}$.

\section{Expired Gas Analysis}

An Expired Gas Analyzer Model AE-280 (Minato Medical Science) was used. Using the breath-by-breath method, the volume of oxygen uptake, volume of carbon dioxide output, end-expiratory oxygen partial pressure and end-expiratory carbon dioxide partial pressure, tidal volume, respiratory rate and respiratory minute volume minute ventilation were measured. The values of 8 successive respirations were averaged and evened. In the ramp protocol exercise test, expiratory gas analysis parameters such as peak oxygen uptake, AT and RCP were determined. AT was detected by the V-slope method using the results from expired gas analysis ${ }^{17}$ In cases where determinations by the $\mathrm{V}$-slope method were difficult, it was computed from a time-trendgram according to the methods of Wasserman et al ${ }^{14-17}$ at the point where the ventilatory equivalent for oxygen started to increase without increase in the ventilatory equivalent for carbon dioxide, the point of an acute increase of the gas exchange ratio and the point where end tidal oxygen started to increase without end tidal carbon dioxide decrease. RCP was determined from the time-trendgram at the point where equivalent for carbon dioxide started to increase and the point when end tidal carbon dioxide started to decrease.

\section{Exercise Protocols}

Exercise tests were conducted using an upright electromagnetically braked cycle ergometer (Corival WLP-400, Lode). Expired gas analysis and the assessment of the deoxygenation of the right vastus lateralis muscle by NIR spectroscopy were performed simultaneously. The blood pressure was measured by a sphygmometer at $1 \mathrm{~min}$ interval. ECG was monitored continuously using a Case 15 Stress System (Marquette Electronics). Each exercise test was carried out $2-3 \mathrm{~h}$ after the last meal and was followed by resting period on the ergometer for at least 4 min until a respiratory steady state was established. At the end of the exercise, the recovery state was observed for $5 \mathrm{~min}$.

Ramp Protocol Exercise All subjects performed a symptom-limited increasing work rate exercise test in a ramp pattern. After a warm-up period of $4 \mathrm{~min}$ at $20 \mathrm{~W}$, an 
increasing work rate at a slope of $10 \mathrm{~W} / \mathrm{min}$ (for heart failure patients), 15 or $20 \mathrm{~W} / \mathrm{min}$ (for sedentary normal subjects) or $30 \mathrm{~W} / \mathrm{min}$ (for active normal subjects) was applied depending on the activity level, weight, sex, age and clinical condition of the subject. The subjects were instructed to maintain the ergometer at $60 \mathrm{rpm}$ during the warm-up and the work rate increase. Although the exercise test was in principle symptom-limited, the test was terminated when a revolution rate of $50 \mathrm{rpm}$ could not be maintained, when the oxygen uptake reached a plateau or decreased, or when the systolic pressure reached 250 $\mathrm{mmHg}$ or higher in heart failure patients.

To determine the reproducibility of the test, 12 of the 21 sedentary normal subjects ( 10 males and 2 females, age $29 \pm 4$ years, height $168 \pm 4 \mathrm{~cm}$, weight $61 \pm 8 \mathrm{~kg}$ ) performed a second exercise test within 1 week of the first test, using the same protocol as the first test.

Constant Work Rate Exercise A 2-stage 6-min constant work rate test was performed in 5 normal males (age $30 \pm 4$ years, height $167 \pm 3 \mathrm{~cm}$, weight $60 \pm 7 \mathrm{~kg}$ ) using an ergometer at work rate intensities below and above the AT level. The below AT level was at $60 \%$ of the AT level as detected by the ramp protocol exercise test, and the above AT level was at an intensity equivalent to $40 \%$ of that from AT to peak $\dot{\mathrm{V}} \mathrm{O}_{2}$. First the below AT level test was performed. After $20 \mathrm{~min}$ of rest in a seated position and full recovery of heart rate and blood pressure to the pre-exercise levels, the above AT level test was conducted. During each exercise test, the deoxygenation in the right vastus lateralis muscle was monitored by NIR spectroscopy.

\section{Statistical Analysis}

Data are reported as means \pm SD. Expired gas analysis and NIR spectroscopy parameters were compared by Student's paired t-test. When three different groups were compared for ventilatory and NIR spectroscopy parameters, a one-way ANOVA combined with Scheffé's test was used. The correlations between ventilatory and NIR spectroscopy parameters, and reproducibility of NIR spectroscopy parameters were assessed by linear regression analysis. A probability value of $\mathrm{p}<0.05$ (two-tailed) was considered significant.

\section{Results}

\section{Ramp Protocol Exercise}

Fig 1 shows an example of the ramp protocol exercise test in a 29 -year-old male sedentary normal subject. The upper panel shows the expired gas analysis and the lower panel shows the results of oxy-Hb and deoxy- $\mathrm{Hb}$ changes measured by NIR spectroscopy, respectively. $\mathrm{Oxy}-\mathrm{Hb}$ increased during the warm-up period, accompanying the blood volume increase. It then remained stable at a plateau until it began to decrease $5 \mathrm{~min}$ after pedaling started, forming the first inflection point (P1) near the AT. Thereafter, the rate of decrease increased and a second inflection point appeared near the RCP. Deoxy-Hb changes were symmetrical with the oxy-Hb changes, with the same inflection points.

Because P1 and P2 were observed near the AT and RCP, respectively, the 2 inflection points were determined by a standard method for all subjects. For P1, regression analysis was performed on 2 straight lines for the oxy-Hb values between the end of the warm-up and the RCP, and the straight line with the best correlation and intercept were

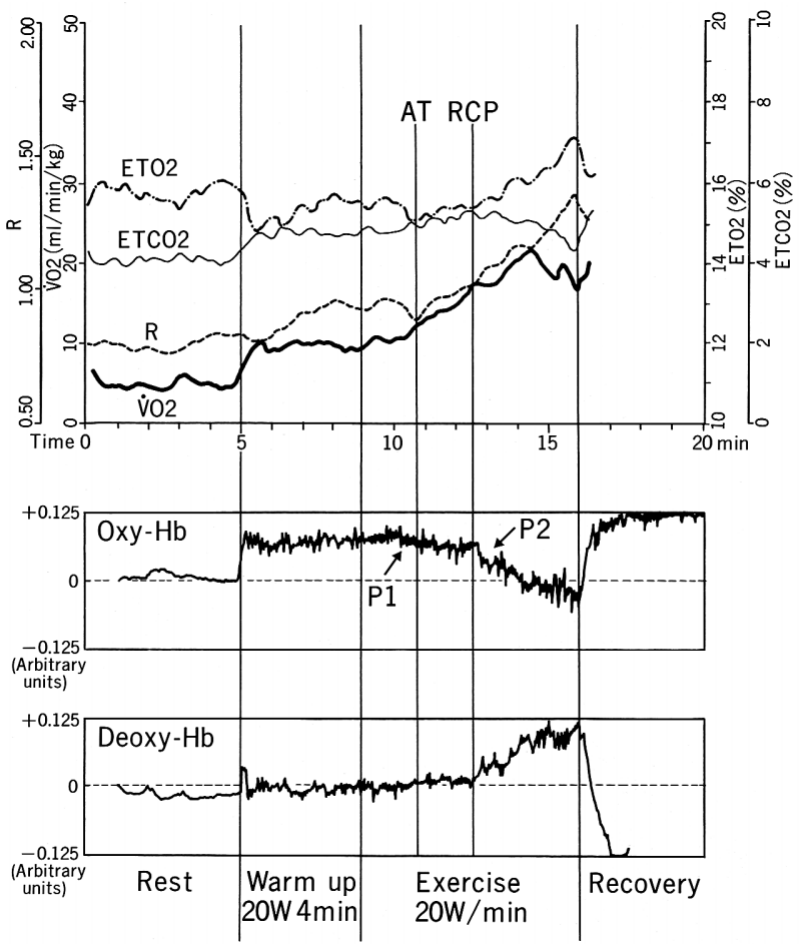

Fig 1. The upper graph shows the expired gas analysis and the lower graph the oxygenated hemoglobin (oxy-Hb) and deoxygenated hemoglobin (deoxy-Hb) measurements done by near-infrared spectroscopy during a ramp protocol exercise in a 29 -year-old healthy male. Oxy$\mathrm{Hb}$ increased when pedaling first commenced and then stayed constant. Near the anaerobic threshold (AT), oxy-Hb started to decrease forming the first inflection point (P1). Near the respiratory compensation point (RCP), the decreasing rate of oxy-Hb was enhanced forming the second inflection point (P2). Deoxy-Hb showed almost symmetrical changes to oxy- $\mathrm{Hb}$, and inflection points were identified as with oxy-Hb. ETO2, end tidal oxygen concentration; $\mathrm{ETCO}_{2}$, end tidal carbon dioxide concentration; R, gas exchange ratio; $\mathrm{VO}_{2}$, oxygen uptake.

selected manually (Fig 2). Similarly, P2 was determined by performing regression analysis on 2 straight lines for the values from the AT to the end of loading, and the best fitted line and intercept were selected.

In the case shown in Fig 1, P2 was formed by the acceleration of the oxy-Hb decrease rate, but in some cases, $\mathrm{P} 2$ was formed by an attenuated oxy-Hb decrease rate (Table 1). When oxy-Hb changes were not clear due to changes in total- $\mathrm{Hb}$, the inflection points were determined from the deoxy-Hb data. In all 43 cases in which AT determination was possible, P1 was identified near the AT. RCP determination was possible in 41 cases, and P2 was identified in 34 cases. The RCP was identified in all the subjects in whom P2 determination was possible, except for one heart failure patient. Both RCP and P2 were determined in 33 subjects (5 active normal subjects, 17 sedentary normal subjects and 11 heart failure patients) (Tables 1 and 2), and P2 was observed near the RCP.

In the experiment on method reproducibility in 12 sedentary normal subjects. AT, P1 and RCP were determined in both tests in all subjects, and P2 was detected in both tests in 9 subjects. A positive correlation was detected between the P1 values obtained in the first and second test $(\mathrm{r}=0.847, \mathrm{p}<0.001) \quad($ Fig $3 \mathrm{~A})$, and between $\mathrm{P} 2$ values obtained in the two tests $(r=0.667, p<0.05)(F i g 3 B)$. When the differences between the values obtained in the first and 


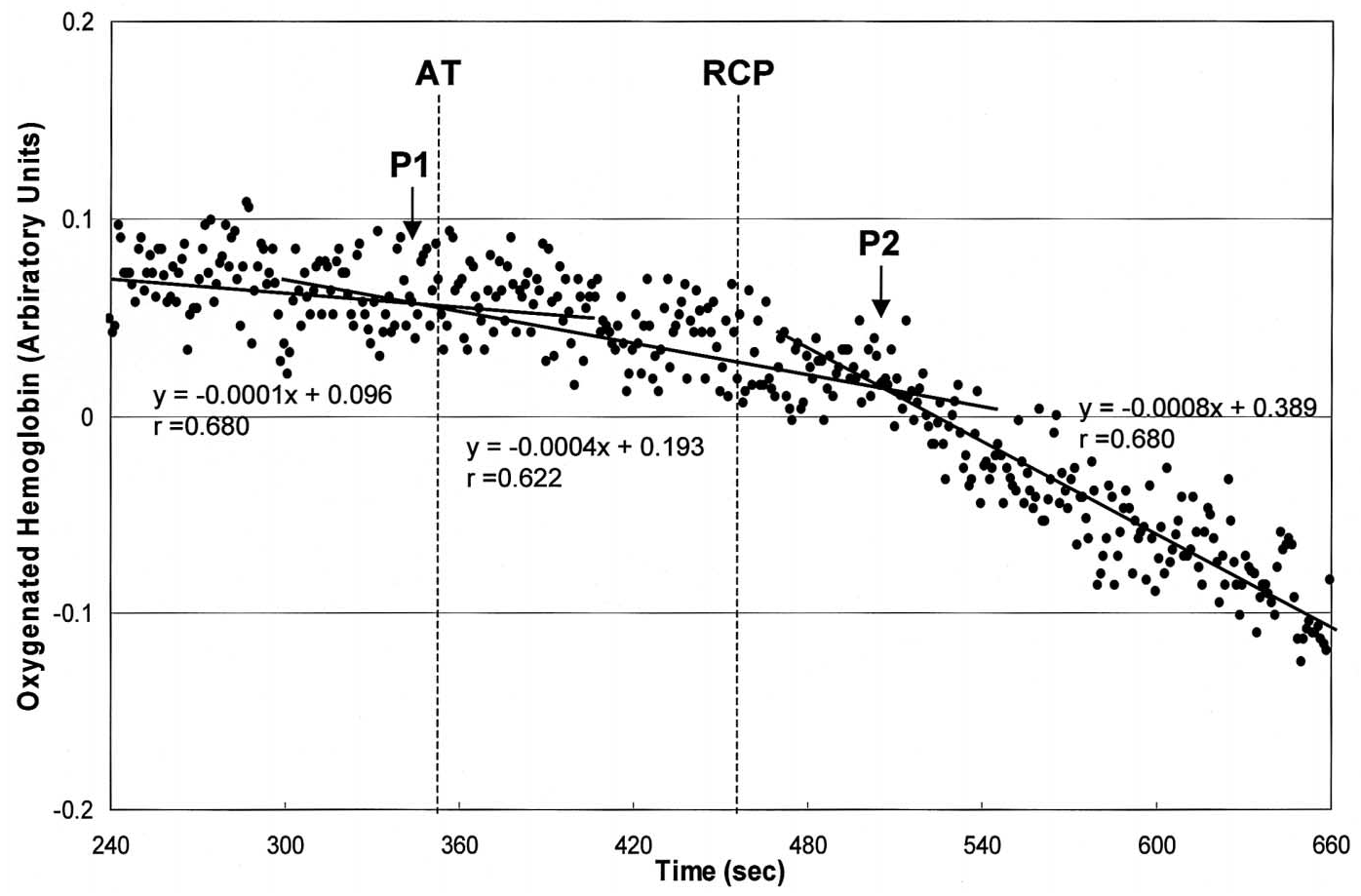

Fig 2. Graph shows determination of the first inflection point (P1) and the second inflection point (P2) during a ramp protocol exercise in the same patient shown in Fig 1. For P1, regression analysis was performed on 2 straight lines for the oxy-Hb values between the end of the warm-up $(240 \mathrm{sec})$ and the respiratory compensation point (RCP), and the straight line with the best correlation and intercept were selected. P2 was determined by performing regression analysis on 2 straight lines for the values from the anaerobic threshold (AT) to the end of loading (660 sec), and the best fitted line and intercept were selected.

Table 1 The Formation Pattern of Second Inflection Points

\begin{tabular}{|c|c|c|c|c|}
\hline & \multicolumn{2}{|c|}{ Normal subjects } & \multirow[b]{2}{*}{$\begin{array}{l}\text { Patients with heart failure } \\
\qquad(n=16)\end{array}$} & \multirow[b]{2}{*}{$\begin{array}{l}\text { All subjects } \\
\quad(n=43)\end{array}$} \\
\hline & $\begin{array}{l}\text { Active } \\
(n=6)\end{array}$ & $\begin{array}{l}\text { Sedentary } \\
(n=21)\end{array}$ & & \\
\hline Accelerated the decrease rate & 4 & 3 & 7 & 14 \\
\hline Attenuated the decrease rate & 1 & 14 & 5 & 20 \\
\hline Not determined & 1 & 4 & 4 & 9 \\
\hline
\end{tabular}

Table 2 Near-infrared Spectroscopy Parameters and Expired Gas analysis Parameters in Ramp Exercise

\begin{tabular}{|c|c|c|c|c|}
\hline & \multicolumn{2}{|c|}{ Normal subjects } & \multirow[b]{2}{*}{$\begin{array}{l}\text { Patients with heart failure } \\
\qquad(n=16)\end{array}$} & \multirow[b]{2}{*}{$\begin{array}{c}\text { All subjects } \\
(n=43)\end{array}$} \\
\hline & $\begin{array}{l}\text { Active } \\
(n=6)\end{array}$ & $\begin{array}{c}\text { Sedentary } \\
(n=21)\end{array}$ & & \\
\hline Peak $\dot{\mathrm{V}} \mathrm{O}_{2}, \mathrm{ml} / \mathrm{min} / \mathrm{kg}$ & $41.4 \pm 6.0^{+t+4}$ & $27.4 \pm 3.5^{\dagger}$ & $15.6 \pm 2.8$ & $25.0 \pm 9.4$ \\
\hline$A T \dot{V} O_{2}, \mathrm{ml} / \mathrm{min} / \mathrm{kg}$ & $19.5 \pm 2.5^{\dagger}$ & $15.9 \pm 2.5^{\dagger}$ & $10.7 \pm 1.9 *$ & $14.5 \pm 3.9 *$ \\
\hline$P 1 \dot{V} O_{2}, \mathrm{ml} / \mathrm{min} / \mathrm{kg}$ & $17.4 \pm 4.5^{\dagger}$ & $15.1 \pm 1.3^{\dagger}$ & $9.9 \pm 1.8$ & $13.4 \pm 4.2$ \\
\hline$R C P \dot{V} \mathrm{O}_{2}, \mathrm{ml} / \mathrm{min} / \mathrm{kg}$ & $34.1 \pm 2.6^{6+3}$ & $22.7 \pm 3.6^{+}$ & $12.9 \pm 2.6$ & $20.3 \pm 7.7$ \\
\hline $\mathrm{P} 2 \dot{\mathrm{V}} \mathrm{O}_{2}, \mathrm{ml} / \mathrm{min} / \mathrm{kg}$ & $33.1 \pm 3.8^{+7}$ & $21.3 \pm 3.1^{\dagger}$ & $13.3 \pm 2.9$ & $20.2 \pm 7.2$ \\
\hline
\end{tabular}

Values are expressed as means $\pm S D$. Both the respiratory compensation point $(R C P)$ and the second inflection point of oxygenated hemoglobin (P2) values could be determined in a total of 33 subjects (active normal subjects, $n=5$; sedentary normal subjects, $n=17$; Patients with heart failure, $n=11) . \dot{V} O_{2}$, oxygen uptake; AT, anaerobic threshold; P1, first inflection point of oxygenated hemoglobin. ${ }^{*} p<0.05$ vs $P 1,{ }^{*} p<0.0001$ vs patients with heart failure, $* 0.0001$ vs sedentary normal subjects.

second tests were plotted against the corresponding average values according to Bland and Altman ${ }^{23}$ the values were all within the $95 \%$ confidence interval (mean difference 2 standard deviations), indicating good reproducibility (Fig 4A, B).

Table 2 shows the peak oxygen uptake and oxygen uptake at the AT, RCP, P1 and P2. All parameters decreased in magnitude first in the active normal subjects, then in the sedentary normal subjects and finally in the patients with heart failure. No significant differences in AT and P1 values were observed between active normal and sedentary normal subjects. P1 was significantly smaller than the AT in all patients, especially in the heart failure patients. RCP and P2 values were similar and no significant 
A. $\mathrm{P} 1 \mathrm{VO}_{2}$

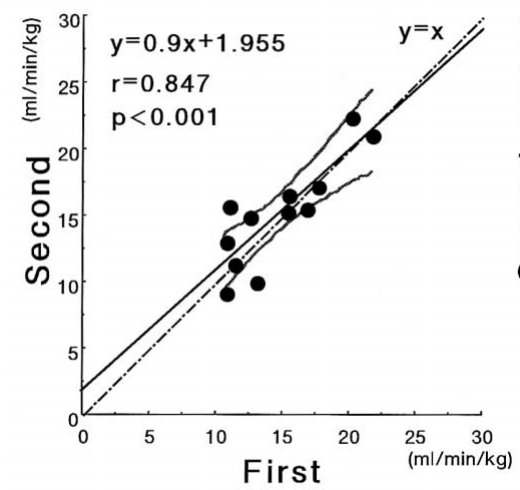

B. $\mathrm{P} 2 \mathrm{~V}_{2}$

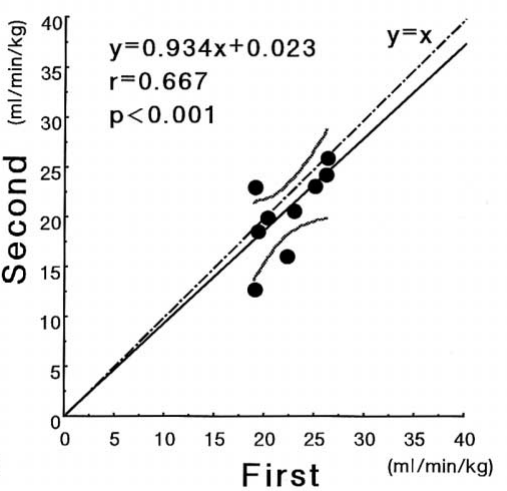

Fig 3. Graphs show the reproducibility of the oxygen uptake $\left(\mathrm{VO}_{2}\right)$ at the first inflection point (P1) (A) and at the second inflection point (P2) (B) in 12 sedentary normal subjects. In the first and second ramp protocol exercise tests, $\mathrm{P} 1$ was determined in all subjects and $\mathrm{P} 2$ was determined in 9 subjects. Both regression lines approximated in a straight line $\mathrm{y}=\mathrm{x} .95 \%$ prediction limits are shown.

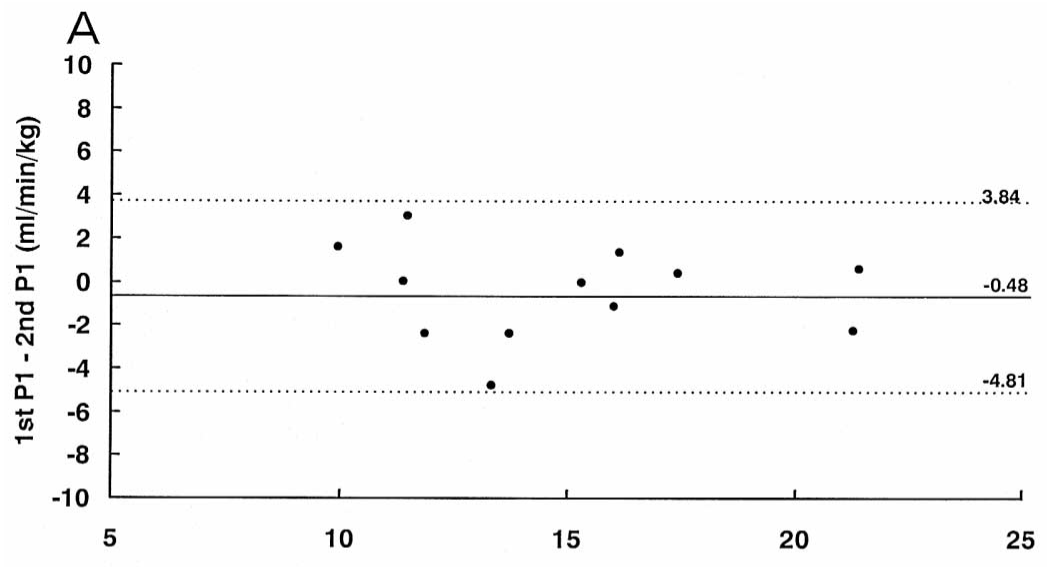

Average 1st $\mathrm{P} 1$ and $2 \mathrm{nd} \mathrm{P1}(\mathrm{ml} / \mathrm{min} / \mathrm{kg})$

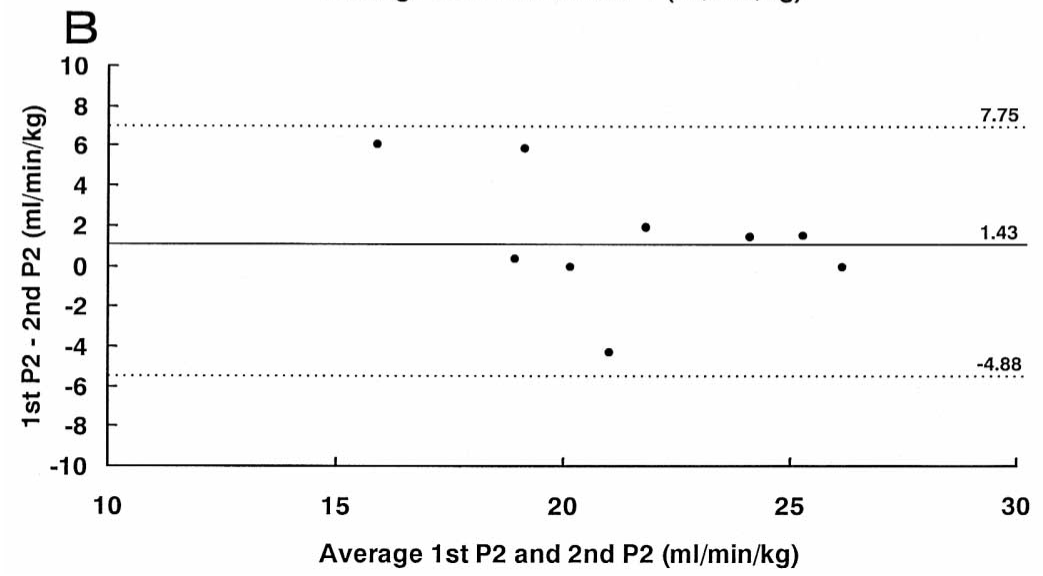

Fig 4. Graphs show the reproducibility of the oxygen uptake $\left(\mathrm{VO}_{2}\right)$ at the first inflection point (P1) (A) and at the second inflection point (P2) (B) in 12 sedentary normal subjects. In the first and second ramp protocol exercise tests, P1 was determined in all subjects and P2 was determined in 9 subjects. The differences between the values obtained in the first and second tests were plotted against the corresponding average values, the values were all within the $95 \%$ confidence interval (mean difference 2 standard deviations). differences in the 2 values were detected between the 3 groups.

Both P1 and P2 values were high in active normal subjects and low in patients with heart failure, indicating that both are indicators of exercise tolerance. A significant high correlation was detected between peak $\dot{\mathrm{V}} \mathrm{O}_{2}$ and $\mathrm{P} 1$ $\dot{\mathrm{V}}{ }_{2}(\mathrm{r}=0.645, \mathrm{p}<0.0005)($ Fig $5 \mathrm{~A})$, and between peak $\dot{\mathrm{VO}}_{2}$ and $\mathrm{P} 2 \dot{\mathrm{V}} \mathrm{O}_{2}(\mathrm{r}=0.899, \mathrm{p}<0.0005)$ (Fig 5B). P1 was found near the AT, and P2 near the RCP. A significant high correlation was observed between AT and P1 ( $\mathrm{r}=0.753$, $0<0.0005$ ) (Fig 6A) and also between RCP and P2 $(\mathrm{r}=0.925, \mathrm{p}<0.0005)($ Fig 6B).

\section{Constant Work Rate Exercise}

Fig 7 shows an example of the constant rate exercise test. Below the AT work rate exercise $(40 \mathrm{~W})$, the $\dot{\mathrm{VO}}_{2}$ reached a steady state and both oxy-Hb and deoxy-Hb were almost unchanged (Fig 7A). Above the AT work rate exercise (146 $\mathrm{W}), \dot{\mathrm{V}} \mathrm{O}_{2}$ continued to increase and did not attain a steady state, while oxy-Hb decreased and deoxy-Hb increased (Fig 7B). Therefore, deoxygenation in the working muscles did not occur at exercise intensities lower than the AT level, but occurred in high intensity exercise. The same results were obtained in all 5 subjects. 
A

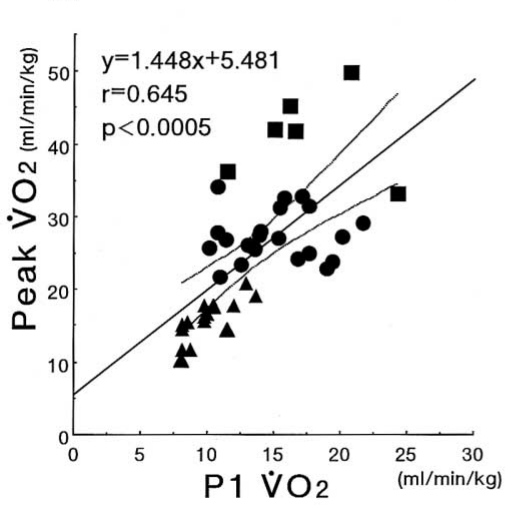

A

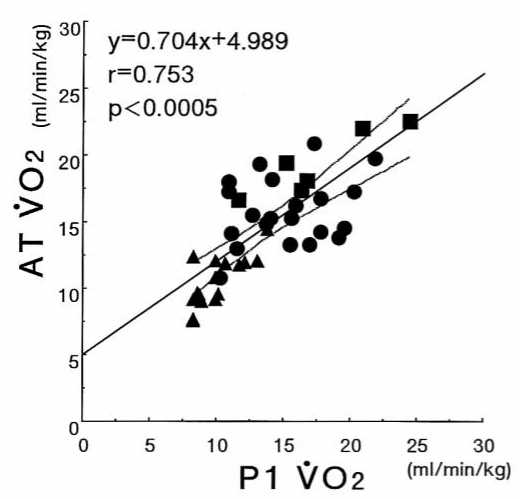

B

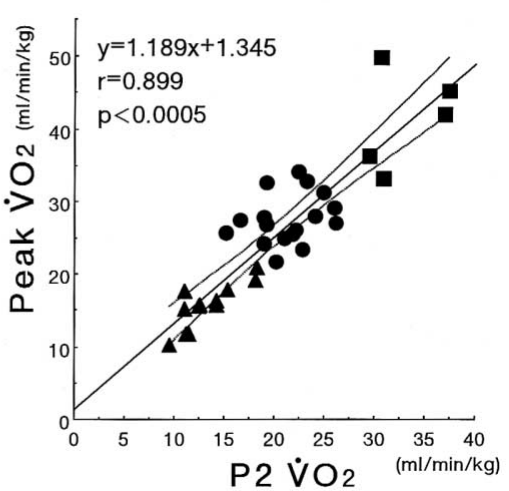

B

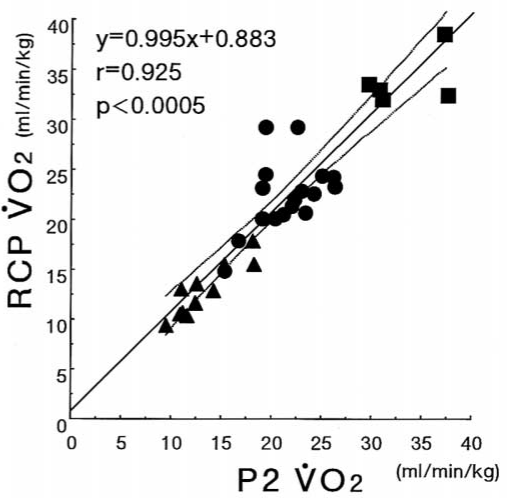

Fig 5. Graphs (A, B) showing relation between the peak oxygen uptake (VO2) with $\mathrm{VO}_{2}$ at the first inflection point (P1) and at the second inflection point (P2). (ם) Active normal subjects (A: $n=6, B: n=6) ;(0)$ sedentary normal subjects (A: $n=21, \quad B: n=17) ;(\boldsymbol{\Delta})$ patients with heart failure $(\mathrm{A}: \mathrm{n}=16, \mathrm{~B}$ : $\mathrm{n}=12$ ). $95 \%$ prediction limits are shown.

Fig 6. Graphs (A, B) showing relation between $\dot{\mathrm{VO}}^{2}$ at the anaerobic threshold (AT) and at P1 and between $\dot{\mathrm{VO}} 2$ at the respiratory compensation point (RCP) and at P2. (घ) Active normal subjects (A: $n=6, B: n=5)$; ( ) sedentary normal subjects $(A: n=21, B$ : $\mathrm{n}=17)$; (A) patients with heart failure (A: $n=16, B: n=11) .95 \%$ prediction limits are shown.

A. Below AT

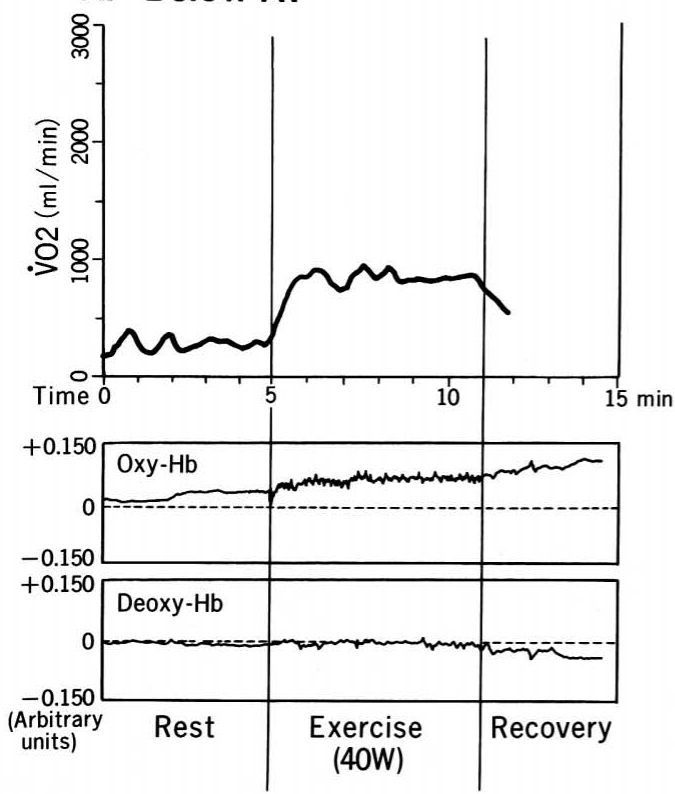

B. Above AT

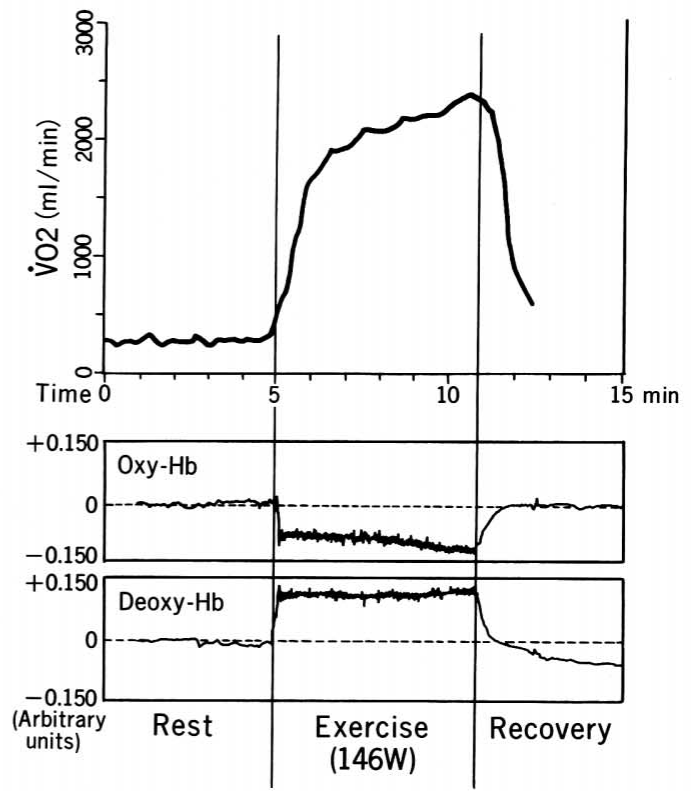

Fig 7. Two different constant work rate exercise tests in a 32-year-old healthy male. Below the anaerobic threshold (AT) work rate, the oxygen uptake $\left(\dot{\mathrm{VO}}_{2}\right)$ reached a steady state and both oxygenated hemoglobin (oxy-Hb) and deoxygenated hemoglobin (deoxy-Hb) were almost unchanged (A). Above the AT work rate, $\dot{\mathrm{V}} \mathrm{O}_{2}$ continued to increase and did not attain a steady state, while oxy-Hb decreased and deoxy-Hb increased (B).

\section{Discussion}

NIR spectroscopy enables continuous non-invasive assessment of the oxygenation-deoxygenation state in working muscles during exercise. Two inflection points (P1 and $\mathrm{P} 2$ ) were detected in the oxy-Hb curve during an 
A. Time-tredgram

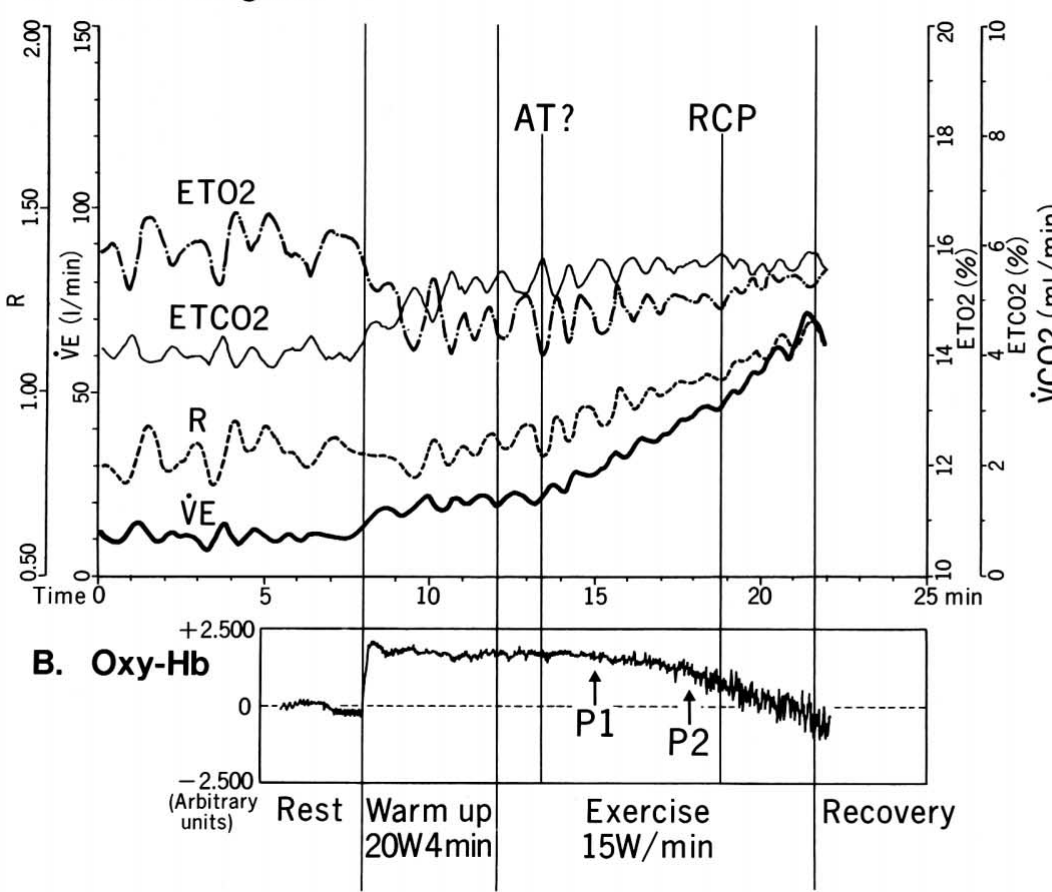

C. V-Slope Method

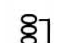

8.

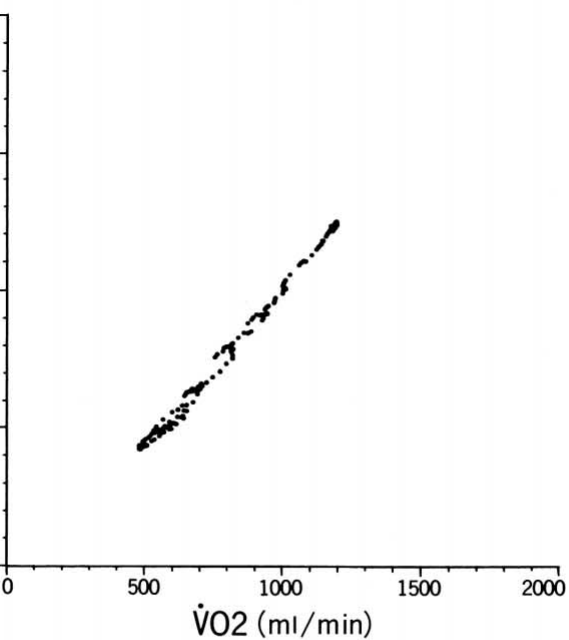

Fig 8. Graphs demonstrate an example of oscillatory ventilation during ramp protocol exercise in a 53-year-old male patient with chronic atrial fibrillation. From the expired gas analysis data, AT was difficult to determine either by the V-slope (B) or the time-trendgram method (A). However, P1 and P2 could be determined from the changes of oxygenated hemoglobin (oxy-Hb) (C). AT, anaerobic threshold; RCP, respiratory compensation point; ETO2, end tidal oxygen concentration; ETCO2, end tidal carbon dioxide concentration; R, gas exchange ratio, VE, minute ventilation; $\dot{\mathrm{V}} \mathrm{O}_{2}$, oxygen uptake; $\dot{\mathrm{V}} \mathrm{CO}$, carbon dioxide output.

increasing work rate exercise, and the reproducibility of these findings was confirmed. Like the AT, RCP and peak oxygen uptake, P1 and P2 values were low in patients with heart failure, and correlated positively with exercise tolerance. A positive correlation was observed between P1 and $\mathrm{AT}$ values as well as P2 and RCP values.

\section{Generalization of the NIR Spectroscopy Parameters}

Although several previous studies reported exercise tests using a sitting ergometer without describing inflection points for oxy-Hb and deoxy-Hb changes in working muscles of the lower limbs, 10,24,25 Belardinelli et al ${ }^{11}$ and Takahashi et al ${ }^{13}$ demonstrated the point at which oxy-Hb started to decrease, and its high correlation with the AT. Honma et al determined the AT in 6 of 10 normal male subjects, and although they did not detect a relationship between oxy-Hb decrease and AT, they observed an oxy$\mathrm{Hb}$ decrease near the AT in 3 cases 26 We designated the point of definite oxy-Hb decrease or deoxy-Hb increase 'P1', which signifies deoxygenation of the working muscles during exercise. P1 was demonstrated in all of the subjects in whom the AT determination was possible using expired gas analysis. Furthermore, the reproducibility of this determination was confirmed. Therefore, the inflection point that indicates metabolic change in working muscles during exercise can be measured if the conditions of measurement are fulfilled. In the present study, inflection points were also determined from deoxy-Hb changes as reference, which further facilitated inflection point determination and increased reliability. With a protocol of progressive load increase of $20 \mathrm{~W}$ per $2 \mathrm{~min}$ or $25 \mathrm{~W}$ per $3 \mathrm{~min}$ as used in previous reports, $8,24,25$ the large change in oxy-Hb during stage-up may obscure the oxy-Hb inflection point. A ramp load should be used to determine the inflection points, as for the AT determination.

\section{Mechanism of Correlation Between Expired Gas Analysis Parameters and NIR Spectroscopy Parameters}

According to Wasserman et al ${ }^{14-16}$ and Beaver et al, ${ }^{17}$ when increased exercise intensity causes an oxygen deficit in working muscles with recruitment of anaerobic metabolism, lactic acid is accumulated which is buffered by bicarbonate ions inside the cell. Increase in intracellular carbon dioxide leads to increase in carbon dioxide in the blood. These changes are reflected in expired gas analysis through ventilation, and the point of initiation of anaerobic metabolism is defined as the AT.

However, the amounts of oxy-Hb and deoxy-Hb are thought to change according to blood volume and the oxyhemoglobin dissociation curve. Recruitment of anaerobic metabolism and the subsequent changes, such as lactic acid accumulation, carbon dioxide increase and hydrogen ion increase due to the Bohr effect, cause a shift to the right of the oxyhemoglobin dissociation curve and enhance the dissociation of oxygen from hemoglobin. These are important factors that lead to oxy-Hb decrease and deoxy-Hb increase. Although P1 and AT almost coincided, P1 tended to occur earlier than the AT. Although this may be explained by the fact that P1 more directly measures the changes in working muscles, the AT and P1 may be regarded as indicators of the initiation of anaerobic metabolism measured from 2 different angles. Our findings of P1 and the AT in close proximity and a good correlation between the 2 parameters support this hypothesis. If this hypothesis is true, then oxy-Hb decrease should be absent in a constant rate exercise test at an intensity lower than the 
AT, but the decrease should occur at an exercise intensity higher than the AT. The results of the constant rate exercise tests in 5 normal subjects supported this hypothesis. Belardinelli et al reported similar findings ${ }^{11}$

Stringer et al performed exercise tests with progressive rate increase of 25 to $40 \mathrm{~W} / \mathrm{min}$ in 5 subjects, and exercise tests with two-stage constant rate increase at intensities lower and higher than the lactic acid threshold in another 5 normal subjects? ${ }^{27}$ They reported that in the femoral vein, the lactic acid level did not increase until the oxygen partial pressure dropped to the lowest level $(20 \mathrm{mmHg})$, and concluded that oxyhemoglobin dissociation above the lactic acid threshold was a direct result of lactic acidosis, or the Bohr effect. These findings are in accordance with our results.

The RCP is the starting point of respiratory compensation in response to exercise-induced metabolic acidosis. Beyond the RCP, the marked increase of hydrogen ions enhances a shift to the right of the oxyhemoglobin dissociation curve and increases oxygen dissociation. The formation of P2 may be explained by the enhanced oxy- $\mathrm{Hb}$ decrease. Myoglobin has an oxygen association power approximately 200 times that of hemoglobin, therefore, it is probably not concerned with low and medium intensity workload, but may have an association with high intensity workload. Although our instrument cannot measure hemoglobin and myoglobin separately, increased deoxygenated myoglobin level during exercise in small muscle group has been reported ${ }^{28}$ In the case of $\mathrm{P} 2$ formation due to enhanced oxy-Hb decrease, this formation may be caused by dissociation of oxygen from myoglobin. However, as P2 may also be formed by an attenuated oxy-Hb decrease (Table 1), this phenomenon requires further study. P2 was formed at high intensity exercise. Although loading was stopped when a revolution rate of $50 \mathrm{rpm}$ could not be maintained, $\mathrm{P} 2$ could still be demonstrated despite an insufficient workload.

\section{Clinical Applications}

$\mathrm{P} 1$ and $\mathrm{P} 2$ are both indicators related to exercise tolerance, and their reproducibility has been confirmed. These parameters may be used to assess disease severity working capacity in heart failure patients, and used to observe changes with time.

Until now, anaerobic metabolism in peripheral skeletal muscles has been assessed by the AT. However, patients with heart failure show oscillatory ventilation and other ventilation problems $, 29,30$ and AT determination from expired gas analysis is difficult in some cases. In the present study, 3 patients with heart failure were excluded from the study, because although P1 was identified, AT determination was difficult due to oscillatory ventilation, irregular respiration and low exercise tolerance. Fig 8 shows an example of oscillatory ventilation in a patient with chronic atrial fibrillation. AT determination from expired gas analysis was difficult by the V-slope method or time-trendgram. However, from the curve of oxy- $\mathrm{Hb}$ changes measured by NIR spectroscopy, P1 and P2 could be determined as intercepts of the regression lines. Therefore, in cases showing oscillatory ventilation, NIR spectroscopy provides an alternative method of assessment by detecting anaerobic metabolism from a different angle. Even in patients in whom AT determination from expired gas analysis is difficult, anaerobic metabolism may be assessed using NIR spectroscopy. However, NIR spec- troscopy could not measure ventilatory parameters, it is necessary concomitant use of NIR spectroscopy and expired gas analysis.

\section{Conclusion}

NIR spectroscopy enables non-invasive assessment of the oxygenation-deoxygenation state of working muscles during exercise, and can also be used to evaluate exercise tolerance and disease severity in patients with heart failure. The NIR spectroscopy parameters are reproducible. Observation of time-related changes in patients with heart failure may be used to evaluate treatment or training effect. By assessing the ventilation state by expired gas analysis and the oxygenation-deoxygenation state of working muscles by NIR spectroscopy, the factors that determine exercise tolerance can be simultaneously assessed in detail.

\section{Acknowledgments}

The authors wish to thank Professor J. P. Barron for his helpful comments on the manuscript.

\section{References}

1. Cohn JN, Johnson GR, Shabetai R, Loeb H, Tristani F, Rector T et al: Ejection fraction, peak exercise oxygen consumption, cardiac ratio, ventricular arrhythmias, and plasma norepinephrine as determinants of prognosis in heart failure. Circulation 1993; 87(Suppl VI): VI-5-VI-16

2. Myers J, Froelicher VF: Hemodynamic determinates of exercise capacity in chronic heart failure. Ann Intern Med 1991; 115: $377-$ 386

3. Jorfeldt L, Wahren J: Leg blood flow during exercise in man. Clin Sci 1971; 41: 459-473

4. Whitney RJ: The measurement of volume changes in human limbs. $J$ Physiol 1953; 121: $1-27$

5. Cerrtelli P, Marconi C, Pendergast D, Meyer M, Heisler N, Piiper J: Blood flow in exercising muscles by xenon clearance and by microsphere trapping. J Appl Physiol 1984; 56: 24-30

6. Jöbsis FF: Noninvasive, infrared monitoring of cerebral and myocardial oxygen sufficiency and circulatory parameters. Science 1977; 198: $1264-1267$

7. Tamura M, Oshino N, Chance B, Silver LA: Optical measurement of intracellular oxygen concentration of rat heart in vitro. Arch Biochem Biophys 1978; 191: 8-22

8. Wilson JR, Mancini DM, McCully K, Ferraro N, Lanoce V, Chance $\mathrm{B}$ : Noninvasive detection of skeletal muscle underperfusion with near-infrared spectroscopy in patients with heart failure. Circulation 1989; 80: $1668-1674$

9. Mancini DM, Farrell L, Wilson JR: Validation of near-infrared spectroscopy in man (abstract). Circulation 1992; 86(Suppl I): I-401

10. Matsui S, Tamura N, Hirakawa T, Kobayashi S, Takekoshi N, Murakami E: Assessment of working skeletal muscle oxygenation in patients with chronic heart failure. Am Heart J 1995; 129: 690-695

11. Belardinelli R, Barstow TJ, Porszasz J, Wasserman K: Skeletal muscle oxygenation during constant work rate exercise. Med Sci Sports Exerc 1995; 27: 512-519

12. Belardinelli R, Barstow TJ, Porszasz J, Wasserman K: Changes in skeletal muscle oxygenation during incremental exercise measured with near infrared spectroscopy. Eur J Appl Physiol 1995; 70: 487492

13. Takahashi M, Yamada T, Kinoshita M: The changes of oxygenated hemoglobin and myoglobin in exercising muscle in patients with heart failure (abstract). Jpn Circ J 1993; 57(Suppl I): I-316 (in Japanese)

14. Wasserman K, McIlroy MB: Detecting the threshold of anaerobic Metabolism metabolism in cardiac patients. Am J Cardiol 1964; 14: $844-852$

15. Wasserman K, Whipp BJ, Koyal SN, Beaver WL: Anaerobic threshold and respiratory gas exchange during exercise. J Appl Physiol 1973; 35: $236-243$

16. Wasserman K, Whipp BJ, Davis JA: Respiratory physiology of exercise: Metabolism, gas exchange, and ventilatory control. Int Rev Physiol III 1981; 23: 149-211

17. Beaver WL, Wasserman K, Whipp BJ: A new method for detecting 
anaerobic threshold by gas exchange. J Appl Physiol 1986; 60: 2020-2027

18. Tamura M: Non-invasive monitoring of brain oxygen metabolism during cardiopulmonary bypass by near-infrared spectrophotometry. Jpn Circ J 1991; 55: 330-335

19. Hazeki O, Tamura M: Quantitative analysis of hemoglobin oxygenation state of rat brain in situ by near-infrared spectrophotometry. $J$ Appl Physiol 1988; 64: 796-802

20. Seiyama A, Hazeki O, Tamura M: Noninvasive quantitative analysis of blood oxygenation in rat skeletal muscle. J Biochem 1988; 103: $419-424$

21. Depley DT, Cope M, Zee P, Arridge S, Wray S, Wyatt J: Estimation of optical pathlength through tissue from direct time of flight measurement. Phys Med Biol 1988; 33: 1433-1442

22. Chance B, Maris M, Sorge J, Zhang MZ: A phase modulation system for dual wavelength difference spectroscopy of hemoglobin deoxygenation in tissues. Proc Soc Photo Optical Instrum Engr 1990; 1204: $481-491$

23. Bland M, Altman DG: Statistical methods for assessing agreement between two methods of clinical measurements. Lancet 1986; 1: $307-310$

24. Mancini DM, Ferraro N, Nazzaro D, Chance B, Wilson JR: Respiratory muscle deoxygenation during exercise in patients with heart failure demonstrated with near-infrared spectroscopy. $J$ Am Coll Cardiol 1991; 18: 492-498

25. Marzo KP, Herrmann HC, Mancini DM: Effect of balloon mitral valvuloplasty on exercise capacity, ventilation and skeletal muscle oxygenation. J Am Coll Cardiol 1993; 21: 856-865

26. Homma S, Fukuoka H, Fujii N, Eda H, Ikegami H: Study of human muscle circulation during bicycle exercise using near-infrared spectroscopy. Jpn J Phys Fitness Sports Med 1992; 41: 586-594 (in Japanese with English abstract)

27. Stringer W, Wasserman K, Casaburi R, Porszasz J, Maehara K, French W: Lactic acidosis as a facilitator of oxyhemoglobin dissociation during exercise. J Appl Physiol 1994; 76: 1462-1467

28. Mancini DM, Wilson JR, Bolinger L, Li H, Kendrick K, Chance B: In vivo magnetic resonance spectroscopy measurement of deoxymyoglobin during exercise in patients with heart failure. Circulation 1994; 99: 500-508

29. Ishizaka S, Asanoi H, Kameyama T, Miyagi K, Sasayama S: Exercise induced oscillatory ventilation in chronic heart failure (abstract). Jpn Circ J 1989; 53: 825-826

30. Miyagi K, Asanoi H, Ishizaka S, Kameyama T, Sasayama S: Limited value of anaerobic threshold for assessing functional capacity in patients with heart failure. Clin Cardiol 1993; 16: 133-137 